Pacific Journal of Mathematics

A CHARACTERIZATION OF EXTREMALS FOR GENERAL

G. W. KimBL 


\title{
A CHARACTERIZATION OF EXTREMALS FOR GENERAL MULTIPLE INTEGRAL PROBLEMS
}

\author{
G. W. KImBLE
}

1. Introduction. The theory of quadratic functionals on a Hilbert space is applied here to variational problems for multiple integrals, involving several dependent variables and their partial derivatives up to any finite order, in nonparametric form. The treatment is confined to integration over open sets with fixed boundary, and to weak neighborhoods of an extremal. The basic result is the establishment of general sufficiency theorems for a weak relative minimum with isoperimetric or differential side conditions. A specific application is made of the sufficiency theorem in the case of isoperimetric side conditions to the extension of a well known characterization of of extremals. This characterization has been treated by Poincaré [16], Birkhoff and Hestenes [1], Karush [9] and others. It is analogous to the characterization of saddle points on two-dimensional surfaces as constrained extrema.

2. Quadratic forms. The theory of quadratic functionals (forms) has been developed explicitly by Hestenes [6,7], and implicitly by Van Hove [19] and writers on elliptic partial differential equations.

If $Q(x)$ is a quadratic form on a Hilbert space $\mathfrak{E}$ with real scalars then there exist unique subspaces $\mathfrak{K}_{+}, \mathfrak{S}_{0}, \mathfrak{S}_{-}$of $\mathfrak{S}_{2}$, having the null vector as their only common element, that are mutually orthogonal and $Q$-orthogonal, are such that $Q$ is positive on $\mathfrak{L}_{+}$, negative on $\mathfrak{S}_{-}$, and zero on $\mathfrak{S}_{0}$, and are such that $\mathfrak{S}_{2}=\mathfrak{S}_{+}+\mathfrak{S}_{0}+\mathfrak{S}_{-}[6$, p. 543]. The sum of the dimensions of the subspaces $\mathfrak{K}_{-}$and $\mathfrak{K}_{0}$ will be called the isoperimetric index of $Q$ on $\mathfrak{S}$. A quadratic form that is representable on $\mathfrak{S}$ as the sum of a positive definite quadratic form and a $w$ continuous quadratic form has been called a Legendre form by M. R. Hestenes. The fact that the isoperimetric index of a Legendre form is finite is significant for the characterization of extremals given below.

If $Q(x)$ and $K(x)$ are quadratic forms on $\mathfrak{S}$ such that $J(b ; x)=$ $Q(x)+b K(x)$ is a Legendre form for every positive number $b, K(x) \leqq 0$, and $Q(x)>0$ whenever $K(x)=0$ and $x \neq 0$, then there is a positive number $c$ such that $J(c ; x)$ is positive definite on $\mathfrak{S}$. A corollary to this is: If $Q(x)$ and $K(x)$ are quadratic forms on $\mathfrak{K}, J\left(b_{0} ; x\right)=$ $Q(x)+b_{0} K(x)$ is a Legendre form for some number $b_{0}, K(x) \geqq 0$, and

Received April 21, 1963, and in revised form November 12, 1963. Revision of doctoral dissertation, University of California, Los Angeles. The work was sponsored in part by the Office of Naval Research and the Office of Ordnance Research, U. S. Army. 
$Q(x)>0$ whenever $K(x)=0$ and $x \neq 0$, then there is a positive number $c \geqq b_{0}$ such that $J(c ; x)$ is positive definite on $\mathfrak{S}$.

Let $\Lambda$ be a metric space. Let $M(\lambda ; x)$ be a real-valued function of $\lambda$ and $x$ on the product space $\Lambda \times \mathfrak{S}$ which has the properties:

(1) for each element $\lambda$ in $A, M(\lambda ; x)$ is continuous in $x$ on $\mathfrak{S}$;

(2) for every $\lambda_{0}$ in $\Lambda, M(\lambda ; x)$ is continuous in $\lambda$ at $\lambda_{0}$ uniformly for all $x$ in $\mathscr{S}$ for which $\|x\|=1$. Then $M(\lambda ; x)$ will be called a $\lambda$ form on $\mathfrak{S}$.

The proofs of the following three theorems are immediate consequences of the above definition:

THEOREM 2.1. If $Q(\lambda ; x)$ is a quadratic form in $x$, then $Q(\lambda ; x)$ is a $\lambda$-form (a quadratic $\lambda$-form) if and only if for every $\varepsilon>0$ and every $\lambda_{0}$ in $\Lambda$ there is a neighborhood $\Lambda_{0}$ of $\lambda_{0}$ such that for $\lambda$ in $\Lambda_{0},\left|Q(\lambda ; x)-Q\left(\lambda_{0} ; x\right)\right| \leqq \varepsilon\|x\|^{2}$.

THEOREM 2.2. If $L(\lambda ; x)$ is a linear form in $x$, then $L(\lambda ; x)$ is $a \lambda$-form (a linear $\lambda$-form) if and only if for every $\varepsilon>0$ and every $\lambda_{0}$ in $\Lambda$ there is a neighborhood $\Lambda_{0}$ of $\lambda_{0}$ such that for $\lambda$ in $\Lambda_{0},\left|L(\lambda ; x)-L\left(\lambda_{0} ; x\right)\right| \leqq \varepsilon\|x\|$.

THEOREM 2.3. If the forms $L_{\sigma}(\lambda ; x)(\sigma=1, \cdots, p)$ are linear $\lambda$ forms, then the form $K(\lambda ; x)=L_{\sigma}(\lambda ; x) L_{\sigma}(\lambda ; x)$ is a quadratic $\lambda$-form.

The sufficiency theorem of $\S 4$ will be based on

THEOREM 2.4. Suppose that $Q(\lambda ; x)$ is a quadratic $\lambda$-form on $\mathfrak{S}$, that $\lambda_{0}$ is in $\Lambda$, and that $\mathscr{K}$ is a subspace of $\mathscr{S}$ on which $Q\left(\lambda_{0} ; x\right)$ is positive definite. Then there is a neighborhood $\Lambda_{0}$ of $\lambda_{0}$ and $a$ positive number $h$ such that for every $\lambda$ in $\Lambda_{0}$, the inequality $Q(\lambda ; x) \geqq$ $h\|x\|^{2}$ holds for all $x$ in $\mathscr{K}$.

Proof. By hypothesis there exists $h>0$ such that for each $x$ in $\mathscr{K}, Q\left(\lambda_{0} ; x\right) \geqq h\|x\|^{2}$. In Theorem 2.1 , choose $\varepsilon=h / 2$; then there is a neighborhood $\Lambda_{0}$ of $\lambda_{0}$ such that

$$
-\frac{h}{2}\|x\|^{2} \leqq Q(\lambda ; x)-Q\left(\lambda_{0} ; x\right) \leqq \frac{h}{2}\|x\|^{2} ;
$$

hence

$$
Q(\lambda ; x) \geqq Q\left(\lambda_{0} ; x\right)-\frac{h}{2}\|x\|^{2} \geqq h\|x\|^{2}-\frac{h}{2}\|x\|^{2}=\frac{h}{2}\|x\|^{2} .
$$

The sufficiency theorem of $\S 5$ will be based on the following 
theorem. Let $Q(\lambda, b ; x)=P(\lambda ; x)+b K(\lambda ; x)$ where $P(\lambda ; x)$ and $K(\lambda ; x)$ are quadratic forms in $x$ defined on $A \times \mathfrak{S}$ and $b$ is a real number. We will assume in the following theorem that for each $\lambda$ in $A, K(\lambda ; x)$ is a nonnegative quadratic form in $x$ on $\mathfrak{K}$, and that for $\lambda=\lambda_{0}$, $P\left(\lambda_{0} ; x\right)>0$ whenever $K\left(\lambda_{0} ; x\right)=0$ and $x \neq 0$.

THEOREM 2.5. If $Q\left(\lambda_{0}, b ; x\right)$ has the property described above and is a Legendre form on $\mathfrak{S}$ for some value of $b$, then there exists a positive number $c$ such that $Q(\lambda, c ; x)$ is positive definite on $\mathfrak{S}$.

Proof. The conclusion is a direct consequence of the corollary given earlier in this section.

3. General multiple integrals. Let $T$ be a bounded open set in an $m$-dimensional euclidean space, and let $t$ be a variable point in $T$. Let $z^{1}, z^{2}, \cdots, z^{n}$ be real-valued functions defined on $T$ and let $z$ represent the set $z^{1}, \cdots, z^{n}$ plus all the partial derivatives, when they exist, of these functions of orders less than or equal to some positive integer $k$. The boundary values of $z$ and the first $k-1$ derivatives are prescribed. Finally, let $f$ denote a real-valued function of $t$ and $z$. With suitable restrictions on the function $f$ and the variable $z$ we can interpret meaningfully the general multiple integral

$$
\int_{T} f[t, z(t)] d t
$$

in the sense of $m$-dimensional Lebesgue integration. Further, let $s$ be another variable point of $T$, let $F$ denote a real-valued function of $t$ and $z$, and let $G$ denote a real-valued function of $s, t, u$, and $v$. The variables $u$ and $v$ are of the same type as $z$. Then the general multiple integral (of Fubini-Tonelli type)

$$
\int_{T} \int_{T} G[s, u(s), t, v(t)] d s d t
$$

is well-defined on the product space $T \times T$ in the same sense as (3.1), as is

$$
J(z)=\int_{T} F[t, z(t)] d t+\int_{T} \int_{T} G[s, z(s), t, z(t)] d s d t .
$$

The treatment of integrals of this type has for one purpose a uniform treatment of variational problems with side conditions.

Partial derivatives will be indicated in the accepted fashion: Given nonnegative integers $\alpha_{1}, \alpha_{2}, \cdots, \alpha_{m}$, let $\alpha=\left(\alpha_{1}, \cdots, \alpha_{m}\right)$ and let $|\alpha|=$ $\alpha_{1}+\alpha_{2}+\cdots+\alpha_{m} ;$ then 


$$
z_{\alpha}^{i}=\frac{\partial^{\prime \alpha \mid} \mid z^{i}}{\partial t_{1}^{\alpha_{1}} \cdots \partial t_{m}^{\alpha_{m}}}
$$

denotes a partial derivative of $z^{i}$ and $j=|\alpha|$ is the order of the derivative. In summary, we have $z=\left[z^{(0)}, z^{(1)}, \cdots, z^{(k)}\right]$ with $z^{(0)}=$ $\left(z^{1}, z^{2}, \cdots, z^{n}\right)$ and $z^{(j)}=\left(z_{a}^{i}\right),|\alpha|=j \geqq 1$.

4. Sufficient conditions for a proper weak relative minimum. Assume as given:

(1) a set $T$ in $t$-space that is a union of a finite number of sets each of which is the image of an $m$-dimensional bounded open interval under a one-to-one continuous transformation which together with its inverse satisfies a uniform Lipschitz condition [7, p. 319];

(2) an open set $\mathscr{R}$ in $t z$-space; let $\mathscr{S}=\mathscr{R} \times \mathscr{R}$;

(3) integrand functions $F(t, z), G(s, u, t, v)$ such that $F$ is continuous on $\mathscr{R}, G$ is continuous on $\mathscr{S}$, and $F$ and $G$ have continuous derivatives on $\mathscr{R}$ and $\mathscr{S}$, respectively, of the first and second orders with respect to the components of $z, u$, and $v$;

(4) a particular function $e$ that is of class $C^{k}$ on the closure of $T,{ }^{1}$ and whose elements $[t, e(t)]$ are in $\mathscr{R}$ for all $t$ in $T$.

A function $z$ belongs to the class $\mathscr{A}$ of comparison functions if

(1) $z$ is of class $C^{k}$ on the closure of $T$;

(2) the difference $z^{(0)}-e^{(0)}$ together with its derivatives of orders $\leqq k-1$ vanish on the boundary of $T$, that is, they have limit functions on the boundary that are identically zero;

(3) the elements $[t, z(t)]$ are in $\mathscr{R}$ for all $t$ in $T$.

The class $\mathscr{B}$ of variations is the closure in the Hilbert space $\mathfrak{F}_{k}$ ([7], p. 319, restricted to real scalars) of the class of $x^{(0)}$ in $\mathfrak{S}_{k}$ whose $k$ th derivatives are bounded on $T$, and which, together with their first $(k-1)$-derivatives, vanish on the boundary of $T$. The boundedness of $T$ ensures that $z-e$ is a variation in the class $\mathscr{B}$ and that the integral $J(z)$ as defined by (3.3) has well-defined values. In what follows the derivatives of $F$ with respect to $z$ and of $G$ with respect to $u$ and $v$ will appear and then their evaluations for the case $z=z(t)$, $u=z(s), v=z(t)$. For all of this it will be convenient to use the abbreviations

$$
\begin{aligned}
& F(t, z)=F[t, z(t)], \quad G(s, t, z)=G[s, z(s), t, z(t)], \\
& G(t, s, z)=G[t, z(t), s, z(s)], \quad F_{z_{\alpha}^{i}}(t, z)=\left[\frac{\partial F(t, z)}{\partial z_{a}^{i}}\right]_{z=z(t)}, \\
& G_{u_{a}^{i}}(s, t, z)=\left[\frac{\partial G(s, t, z)}{\partial u_{a}^{i}}\right]_{\substack{u=z(s) \\
v=z(t)}}, \quad G_{v_{\alpha}^{i}}(s, t, z)=\left[\frac{\partial G(s, t, z)}{\partial v_{a}^{i}}\right]_{\substack{u=z(s) \\
v=z(t)}},
\end{aligned}
$$

1 The function $z$ belongs to class $C^{k}$ if each component of $z^{(0)}$ is in the class. Similar remarks apply to the functions $e, x$, and $y$. 
and similarly for higher derivatives of $F$ and $G$.

The sufficient conditions will be stated in terms of the first and second variations of $J(z)$ : The first variation of $J(z)$ on $e$ is given by

$$
J_{1}(e ; x)=\int_{T}\left\{F_{z_{\alpha}^{i}}(t, e)+\int_{T}\left[G_{u_{\alpha}^{i}}(t, s, e)+G_{v_{\alpha}^{i}}(s, t, e)\right] d s\right\} x_{\alpha}^{i}(t) d t
$$

where $i$ and $\alpha$ are summed for $i \leqq n$ and $|\alpha| \leqq k$. The second variation of $J(z)$ on $e$ is given by

$$
J_{2}(e ; x)=\int_{T} r_{\alpha \beta}^{i j}(t, e) x_{\alpha}^{i}(t) x_{\beta}^{j}(t) d t+\int_{T} \int_{T} w_{\alpha \beta}^{i j}(s, t, e) x_{\alpha}^{i}(s) x_{\beta}^{j}(t) d s d t
$$

(summed on $i, j, \alpha, \beta$ with $i \leqq n, j \leqq n,|\alpha| \leqq k,|\beta| \leqq k$ ) where

$$
r_{\alpha \beta}^{i j}(t, e)=F_{z_{\alpha_{\beta}}^{i} z}(t, e)+\int_{T}\left[G_{u_{\omega_{j}^{i}}^{i} j_{\beta}^{j}}(t, s, e)+G_{v_{\alpha}^{i} v_{\beta}^{j}}(s, t, e)\right] d s
$$

and

$$
w_{\alpha \beta}^{i j}(s, t, e)=G_{u_{\alpha}^{i} v_{\beta}^{j}}(s, t, e)+G_{v_{\alpha}^{i} u j}(s, t, e) .
$$

The function $e$ satisfies Condition I if

$$
J_{1}(e ; x)=0
$$

for every variation in $\mathscr{B}$.

A function $z$ of class $C^{k}$ on the closure of $T$ satisfying I will be called an extremal of $J(z)$.

The function $e$ satisfies Condition III' if there is a positive constant $\varepsilon$ such that

$$
r_{\gamma \delta}^{i j}(t, e) \xi^{\xi} \xi^{\delta} \zeta^{i} \zeta^{j} \geqq \varepsilon|\xi|^{2 k}|\zeta|^{2}
$$

(summed on $i, j, \gamma, \delta$, for $i \leqq n, j \leqq n,|\gamma|=|\delta|=k$ ) for all $t$ in $T$ and all sets of real numbers $(\not \equiv 0) \xi=\left(\xi_{1}, \cdots, \xi_{m}\right), \zeta=\left(\zeta^{1}, \cdots, \zeta^{n}\right)$. The notation $\xi^{\gamma}$ means $\left(\xi_{1}\right)^{\gamma_{1}}\left(\xi_{2}\right)^{\gamma_{2}} \cdots\left(\xi_{m}\right)^{\gamma_{m}}$; also, $|\xi|^{2}=\left(\xi_{1}\right)^{2}+\cdots+\left(\xi_{m}\right)^{2}$ and $|\zeta|^{2}=\left(\zeta^{1}\right)^{2}+\cdots+\left(\zeta^{n}\right)^{2}$. The functions $r_{\gamma \delta}^{i j}(t, z)$ are those given in (4.3). Condition III' has been referred to as the strengthened condition of Legendre.

The function $e$ satisfies Condition IV' if

$$
J_{2}(e ; x)>0
$$

holds for every nonnull variation in $\mathscr{B}$. By a nonnull variation is meant one that does not vanish identically on $T$.

Sufficient conditions similar to these have been established by Van Hove $[18,19]$ for the case of derivatives of the first order only appearing in the integrand, and by Klotzler [11] for higher derivatives but with the assumption that $J_{2}$ is positive definite. 
The sufficiency theorem here uses, in addition to Theorem 2.4, the following three theorems which were proved in [7] as Theorems 6:1 and 8: 1:

(1) The quadratic form $K(x)=\int_{T} b_{\mu \nu}^{i j} x_{\mu}^{i} x_{\nu}^{j} d t$ (summed on $i, j, \mu, \nu$ for $i \leqq n, j \leqq n,|\mu| \leqq k,|\nu| \leqq k,|\mu|+|\nu|<2 k)$, where $b_{\mu \nu}^{i j}(t)=b_{\nu \mu}^{j i}(t)$ are bounded integrable functions of $t$ on $T$, is $w$-continuous on $\mathfrak{S}_{k}$.

(2) The quadratic form $H(x)=\int_{T} \int_{T} c_{\alpha \beta}^{i j}(s, t) x_{\alpha}^{i}(s) x_{\beta}^{j}(t) d s d t$ (summed on $i, j, \alpha, \beta$ for $i \leqq n, j \leqq n,|\alpha| \leqq k,|\beta| \leqq k)$ where $c_{\alpha \beta}^{i j}(s, t)=c_{\beta \alpha}^{j i}(t, s)$ are square integrable functions of $s$ and $t$ on $T \times T$, is $w$-continuous on $\mathfrak{S}_{k}$.

(3) Let $J$ be a quadratic form of the type $J(x)=D(x)+K(x)$ where $K(x)$ is $w$-continuous on $\mathscr{B}$ and

$$
D(x)=\int_{T} d_{\gamma \delta}^{i j} x_{\gamma}^{i} x_{\delta}^{j} d t
$$

(summed over $i, j, \gamma, \delta$ for $|\gamma|=|\delta|=k, i \leqq n, j \leqq n$ ) where $d_{\gamma \delta}^{i j}(t)=$ $d_{\delta \gamma}^{j i}(t)$ are continuous functions of $t$ on the closure of $T$. Then the quadratic form $J$ as described above is a Legendre form on $\mathscr{B}$ if and only if it satisfies on $T$ the inequality

$$
d_{\gamma \delta}^{i j} \xi^{\gamma} \xi^{\delta} \zeta^{i} \zeta^{j} \geqq \varepsilon|\xi|^{2 k}|\zeta|^{2}
$$

(summed on $i, j, \gamma, \delta$ for $i \leqq n, j \leqq n,|\gamma|=|\delta|=k$ ) for some positive $\varepsilon$.

LEMMA 4.1. For the function $e, J_{2}(e ; x)$ is a Legendre form on $\mathscr{B}$ if and only if the Condition III' is satisfied.

Proof. Write, using (4.4), $J_{2}(e ; x)=D(x)+K(x)$ where $D(x)$ is given by $D(x)=\int_{T} r_{\gamma \delta}^{i j} x_{\gamma}^{i} x_{\delta}^{j} d t$ (summed on $\gamma$ and $\delta$ for $|\gamma|=|\delta|=k$ ). The immediately preceding theorems apply.

LEMMA 4.2. For the function $e, J_{2}(e ; x)$ is positive definite on $\mathscr{B}$ if and only if the Conditions III' and IV' are satisfied.

Proof. If $J_{2}$ is positive definite on $\mathscr{B}$, then $J_{2}(e ; x)>0$ holds on $\mathscr{B}$ and also $J_{2}$ is a Legendre form; hence it follows that the inequality (4.6) is satisfied on T. On the other hand suppose $J_{2}$ is positive on $\mathscr{B}$ and (4.6) holds. Then $J_{2}$ is a Legendre form on $\mathscr{B}$ by the preceding lemma, and a positive Legendre form is positive definite.

To apply the theorems of $\S 2$ concerning quadratic forms, it is necessary to specify the metric space $\Delta$. For this we will use the weak neighborhoods of the calculus of variations: Let $\left|z_{\alpha}^{i}-e_{\alpha}^{i}\right|=$ $\max _{t}\left|z_{\alpha}^{i}(t)-e_{\alpha}^{i}(t)\right|,\left|z^{(j)}-e^{(j)}\right|=\max _{i, a}\left|z_{\alpha}^{i}-e_{\alpha}^{i}\right|(|\alpha|=j)$; the metric 
space $\Lambda$ is the class $\mathscr{A}$ with the metric

$$
|z-e|=\left|z^{(0)}-e^{(0)}\right|+\left|z^{(1)}-e^{(1)}\right|+\cdots+\left|z^{(k)}-e^{(k)}\right| \text {. }
$$

LEMmA 4.3. The second variation $J_{2}(z ; x)$ is a quadratic $z$-form.

Proof. Apply Theorem 2.1, recalling that $F$ and $G$ are assumed to be of class $C^{2}$.

THEOREM 4.1. If the function e as given satisfies the Conditions I, III', and IV' then there is a (weak) neighborhood $N$ of e and positive constant $\rho$ such that for every $z$ of class $\mathscr{A}$ in $N$

$$
J(z)-J(e) \geqq \rho\|z-e\|^{2} \text {. }
$$

Proof. For any $z$ in $\mathscr{A}$, form $x=z-e$; then $x$ is a variation in the class $\mathscr{B}$. The continuity properties of $F$ and $G$ make possible the expansion

$$
J(z)-J(e)=\int_{0}^{1}(1-\theta) J_{2}(e+\theta x ; x) d \theta
$$

where we have used the fact that I implies $J_{1}(e ; x)=0$. To apply Theorem 2.4, we note that from Lemma $4.2 J_{2}$ is positive definite on $e$, and from Lemma 4.3 $J_{2}$ is a quadratic $z$-form. Consequently there is a neighborhood $N$ of $e$, which can be chosen to be convex, and a constant $\rho>0$ such that when $z$ is restricted to $N$ and $x=z-e$, $J_{2}(e+\theta x ; x) \geqq 2 \rho\|x\|^{2}$. Hence $J(z)-J(e) \geqq \rho\|z-e\|^{2}$.

5. Isoperimetric side conditions. The preceding sufficiency theorem will be applied to the problem of establishing sufficient conditions that a function $e$ will minimize

$$
I(z)=\int_{T} f[t, z(t)] d t+\int_{T} \int_{T} g[s, z(s), t, z(t)] d s d t
$$

subject to a finite number of isoperimetric side conditions

$$
I^{\sigma}(z)=\int_{T^{\prime}} f^{\sigma}[t, z(t)] d t=0 \quad(\sigma=1, \cdots, p) .
$$

For the complete formulation of the problem, it is expected that the assumptions of $\S 4$ are met. In particular, the integrand functions $f(t, z), f^{\sigma}(t, z)$ and their first and second derivatives are specified to be continuous on $\mathscr{R}$ and similarly for $g(s, t, z)$ on $\mathscr{S}$.

Sufficient conditions for weak and strong minima with isoperimetric side conditions, in the case of one dependent variable and derivatives 
of first order, have been treated by Hestenes [5]. The extension of this work to the general case is still unknown. The following conditions $s^{2}$ will prove to be sufficient that $e$ provides a weak relative minimum for the system (5.1) and (5.2).

$I_{s}$. There exists a set of multipliers $a_{\sigma}$ (possibly all zero) such that

$$
I_{1}(e ; x)+a_{\sigma} I_{1}^{\sigma}(e ; x)=0
$$

for every $x$ in $\mathscr{B}$.

III's. The multipliers in $I_{s}$ can be chosen so that there is a positive constant $\varepsilon$ such that

$$
R_{\gamma \delta}^{i j}(t, e) \xi^{\gamma \xi^{\delta} \zeta^{i} \zeta^{j}} \geqq \varepsilon|\xi|^{2 k}|\zeta|^{2}
$$

(summed on $i, j, \gamma, \delta$ for $i \leqq n,|\gamma|=|\delta|=k, j \leqq n$ ) for all $t$ in $T$. The coefficients $R_{\gamma \delta}^{i j}$ are given by

$$
R_{\gamma \delta}^{i j}(t, z)=f_{z_{\gamma}^{i} z_{\delta}^{j}}(t, z)+a_{\sigma} f_{z_{\gamma}^{i} z_{\delta}^{j}}^{\sigma}(t, z)+\int_{T}\left[g_{u_{\gamma}^{i} u_{\delta}^{j}}(t, s, z)+g_{v_{\gamma}^{i} v_{\delta}^{j}}(s, t, z)\right] d s .
$$

$\mathrm{IV}_{s}^{\prime}$. The multipliers in $\mathrm{III}_{s}^{\prime}$ can be chosen so that

$$
I_{2}(e ; x)+a_{\sigma} I_{2}^{\sigma}(e ; x)>0
$$

for every nonnull variation in the subspace of $\mathscr{B}$ for which

$$
I_{1}^{\sigma}(e ; x)=0 \quad(\sigma=1, \cdots, p) .
$$

To reduce this problem to that of $\S 4$, take for the integrands in (3.3): $\quad F=f+a_{\sigma} f^{\sigma}$ and $G=g+b f^{\rho} f^{\sigma}$ summed on $\sigma$ from 1 to $p$, with $b$ a real number yet to be determined. Then, by Fubini's theorem, $J(z)=I(z)+a_{\sigma} I^{\sigma}(z)+b I^{\sigma}(z) I^{\sigma}(z)$; it is easily verified that

$$
J_{1}(z ; x)=I_{1}(z ; x)+a_{\sigma} I_{1}^{\sigma}(z ; x)+2 b I^{\sigma}(z) I_{1}^{\sigma}(z ; x)
$$

and

$$
J_{2}(z ; x)=I_{2}(z ; x)+a_{\sigma} I_{2}^{\sigma}(z ; x)+2 b I_{1}^{\sigma}(z ; x) I_{1}^{\sigma}(z ; x)+2 b I^{\sigma}(z) I_{2}^{\sigma}(z ; x) .
$$

Condition $\mathrm{I}_{s}$ implies the condition I directly, using the facts that $I_{1}^{\sigma}(z ; x)$ is bounded for each value of $\sigma$, and $I^{\sigma}(e)=0$ : We have on $e$, $J_{1}(e ; x)=I_{1}(e ; x)+a_{\sigma} I_{1}^{\sigma}(e ; x)=0$.

Similarly, we can evaluate the expression in III' from its definition by (4.3), and using again that $I^{\sigma}(e)=0$ it follows that $\mathrm{III}_{s}^{\prime}$ implies III'.

${ }^{2}$ In this and the following sections the subscript $s$ will be used to distinguish sufficient conditions involving side conditions. 
LEMma 5.1. If $\mathrm{III}_{s}^{\prime}$ and $\mathrm{IV}_{s}^{\prime}$ hold, then there is a positive value of $b$ such that $J_{2}(e ; x)$ is a positive definite form on $\mathscr{B}$; hence IV' holds.

Proof. Since $I^{\sigma}(e)=0$ and $I_{2}^{\sigma}(e ; x)$ is bounded for each value of $\sigma$, $J_{2}(e ; x)=I_{2}(e ; x)+a_{\sigma} I_{2}^{\sigma}(e ; x)+2 b I_{1}^{\sigma}(e ; x) I_{1}^{\sigma}(e ; x) . \quad J_{2}(e ; x)$ is a Legendre form on $\mathscr{B}$ by Lemma 4.1. Let $P(x)=I_{2}(e ; x)+a_{\sigma} I_{2}^{\sigma}(e ; x)$ and $K(x)=$ $2 I_{1}^{\sigma}(e ; x) I_{1}^{\sigma}(e ; x)$. Then $K(x)$ is a nonnegative quadratic form in $x$. Also, $K(x)=0$ only if $I_{1}^{\sigma}(e ; x)=0$ for $\sigma=1, \cdots, p$. Condition $I V_{s}^{\prime}$ implies that $P(x)>0$ whenever $K(x)=0$ and $x \neq 0$. Consequently the hypotheses of Theorem 2.5 are satisfied, and a positive value for $b$ exists such that $J_{2}(e ; x)$ is positive definite on $\mathscr{B}$. Since a positive definite form is positive, $I V^{\prime}$ is satisfied.

In view of this last result, Theorem 4.1 has as its interpretation in the case of a finite number of isoperimetric side conditions,

THEOREM 5.1. If the function $e$ as prescribed in the formulation of $\S 4$ satisfies the conditions $\mathrm{I}_{s}$, $\mathrm{III}_{s}^{\prime}$ and $\mathrm{IV}_{s}^{\prime}$, then there is a (weak) neighborhood $N$ of $e$ and positive constants $b$ and $\rho$ such that for every $z$ of class $\mathscr{A}$ in $N$

$$
I(z)-I(e)+a_{\sigma} I^{\sigma}(z)+b I^{\sigma}(z) I^{\sigma}(z) \geqq \rho\|z-e\|^{2}
$$

(summed on $\sigma$ for $\sigma \leqq p$ ).

COROLLARY 5.1. Under the hypotheses of Theorem 5.1, there is $a$ (weak) neighborhood $N$ of $e$ and a positive constant $\rho$ such that for every $z$ of class $\mathscr{A}$ in $N$

$$
I(z)-I(e) \geqq \rho\|z-e\|^{2}
$$

holds whenever $z$ also satisfies the conditions

$$
I^{\sigma}(z)=0
$$$$
(\sigma=1, \cdots, p) \text {. }
$$

6. Differential side conditions. In this section the application of Theorem 4.3 is continued to the problem of establishing sufficient conditions that a function $e$ will minimize the functional $I(z)$ given by (5.1) subject to a finite number of partial differential equations as side conditions of the form

$$
\phi^{\sigma}[t, z(t)]=0
$$$$
(\sigma=1, \cdots, p) \text {. }
$$

In addition to the assumptions of $\S 4$, it is required that, for $\sigma=$ $1,2, \cdots, p, \phi^{\sigma}(t, z)$ is continuous and has continuous first and second derivatives on $\mathscr{R}$ with respect to the components of $z$. Further, to 
avoid an empty class of comparison functions, it will be assumed that the set (6.1) satisfies suitable integrability conditions. For the statement of sufficient conditions for a minimum it is convenient to form

$$
H(z)=I(z)+\int_{T} a_{\sigma}(t) \phi^{\sigma}(t, z) d t
$$

and

$$
P(z)=\int_{T} \phi^{\sigma}(t, z) \phi^{\sigma}(t, z) d t
$$

Then for the first and second variations of $H$ and $P$ we take (with summations on $\sigma, i, j, \alpha, \beta$, for $\sigma \leqq p, i \leqq n, j \leqq n,|\alpha| \leqq k,|\beta| \leqq k)$ $H_{1}(z ; x)=I_{1}(z ; x)+\int_{T} a_{\sigma}(t) \phi_{z_{\alpha}^{i}}^{\sigma}(t, z) x_{\alpha}^{i}(t) d t$,

$$
\begin{aligned}
P_{1}(z ; x)= & 2 \int_{T} \phi^{\sigma}(t, z) \phi_{z_{\alpha}^{i}}^{\sigma}(t, z) x_{\alpha}^{i}(t) d t, \\
H_{2}(z ; x)= & I_{2}(z ; x)+\int_{T} a_{\sigma}(t) \phi_{z_{\alpha}^{i} z_{\beta}^{j}}^{\sigma}(t, z) x_{\alpha}^{i}(t) x_{\beta}^{j}(t) d t, \\
P_{2}(z ; x)= & 2 \int_{T} \phi_{z_{\alpha}^{i}}^{\sigma}(t, z) \phi_{z_{\beta}^{j}}^{\sigma}(t, z) x_{\alpha}^{i}(t) x_{\beta}^{j}(t) d t \\
& +2 \int_{T} \phi^{\sigma}(t, z) \phi_{z_{\alpha}^{i} z_{\beta}^{j}}^{\sigma}(t, z) x_{\alpha}^{i}(t) x_{\beta}^{j}(t) d t .
\end{aligned}
$$

As sufficient conditions for a proper weak relative minimum of $I(z)$ subject to $\phi^{\sigma}(t, z)=0$, consider

$I_{s}$. There exist a set of multipliers $\alpha_{\sigma}(t)(\sigma=1, \cdots, p)$ defined and continuous on the closure of $T$ such that

$$
H_{1}(e ; x)=0
$$

for every $x$ in $\mathscr{B}$.

III's. The multipliers $a_{\sigma}(t)$ in $I_{s}$ can be chosen so that there is a positive constant $\varepsilon$ such that the inequality

$$
R_{\gamma \delta}^{i j}(t, e) \xi \xi^{\gamma} \xi^{\delta} \zeta^{i} \zeta^{j} \geqq \varepsilon|\xi|^{2 k}|\zeta|^{2}
$$

(summed on $i, j, \gamma, \delta$ for $i \leqq n, j \leqq n,|\gamma|=|\delta|=k$ ) holds on $T$ for all sets $(\xi, \zeta) \neq(0,0)$ which satisfy $\phi_{z \gamma}^{\sigma}(t, e) \xi^{\gamma} \zeta^{i}=0(\sigma=1, \cdots, p)$. The coefficients $R_{\gamma \delta}^{i j}$ are given by

$$
R_{\gamma \delta}^{i j}(t, z)=f_{z_{\gamma}^{i} z_{\delta}^{j}}(t, z)+a_{\sigma}(t) \phi_{z_{\gamma}^{i} z_{j}^{j}}^{\sigma}(t, z)+\int_{T}\left[g_{u_{\gamma}^{i} u_{\delta}^{j}}(t, s, z)+g_{v_{\gamma}^{i} v_{\delta}^{j}}(s, t, z)\right] d s .
$$

$\mathrm{IV}_{s}^{\prime}$. The multipliers $a_{\sigma}(t)$ in $\mathrm{III}_{s}^{\prime}$ can be chosen so that the 
inequality

$$
H_{2}(e ; x)>0
$$

holds for every nonnull variation for which $\phi_{z_{\alpha}^{i}}^{\sigma}(t, e) x_{\alpha}^{i}(t)=0(\sigma=1, \cdots, p)$ (summed on $i, \alpha$ for $i \leqq n,|\alpha| \leqq k$ ).

For this application take for the integrands in (3.3): $F=$ $f+a_{\sigma} \phi^{\sigma}+b \phi^{\sigma} \phi^{\sigma}$ (summed on $\sigma$ ) with $b$ an as yet undetermined real number, and $G=g$. Then $J(z)=H(z)+b P(z)$, and for the first and second variations of $J$ we have $J_{1}(z ; x)=H_{1}(z ; x)+b P_{1}(z ; x)$ and $J_{2}(z ; x)=$ $H_{2}(z ; x)+b P_{2}(z ; x)$.

Condition $I_{s}$ implies the Condition I directly, using the fact that $\phi^{\sigma}(t, e)=0$ in $(6.4)$, so that $J_{1}(e ; x)=H_{1}(e ; x)$.

LEMMA 6.1. If $\mathrm{III}_{s}^{\prime}$ holds, then there is a positive value of $b$ and a positive number $\varepsilon$ such that for the arguments $[t, e(t)]$, the inequality

$$
\left(R_{\gamma^{\prime}}^{i j}+b \phi_{z_{\gamma}^{i}}^{\sigma} \phi_{z}^{\sigma}\right) \xi^{\gamma} \xi^{\delta} \zeta^{i} \zeta^{j} \geqq \varepsilon|\xi|^{2 k}|\zeta|^{2}
$$

(summed on $i, j, \sigma, \gamma, \delta$ for $i \leqq n, j \leqq n, \sigma \leqq p,|\gamma|=|\delta|=k$ ) holds for every set $(\xi, \zeta)$. Hence $I I I_{s}^{\prime}$ implies III' for this choice of $b$.

Proof. Let $R=R_{\gamma \delta}^{i j}(t) \xi \gamma \xi^{\delta} \zeta^{i} \zeta^{j}$ and $\phi=\phi_{z_{\gamma}^{i}}^{\tau}(t) \phi_{z_{\delta}^{j}}^{\sigma}(t) \xi^{\gamma} \xi^{\delta} \zeta^{i} \zeta^{j}$, and without loss of generality consider the special case $|\xi|=|\zeta|=1$. Then by $\mathrm{III}_{s}^{\prime}, R \geqq \varepsilon>0$ whenever $\phi=0$ and $(\xi, \zeta) \neq(0,0)$; this means that both $R \leqq 0$ and $\phi=0$ only if $(\xi, \zeta)=(0,0)$. Now suppose no positive value of $b$ exists such that III' holds. Then there is a sequence $\left\{t_{q}, \xi_{q}, \zeta_{q}\right\}$ with $\left|\xi_{q}\right|=\left|\zeta_{q}\right|=1$, convergent to $\left(t_{0}, \xi_{0}, \zeta_{0}\right)$, such that in the limit (because of the continuity of $R$ and $\phi$ in $t, \xi$, and $\zeta$ ) $R \leqq 0$, $\phi=0$, and $\left|\xi_{0}\right|=\left|\zeta_{0}\right|=1$.

LEMMA 6.2. If Conditions $\mathrm{III}_{s}^{\prime}$ and $\mathrm{IV}_{s}^{\prime}$ hold, then there is a positive value of $b$ such that $H_{2}(e ; x)+b P_{2}(e ; x)$ is a positive definite

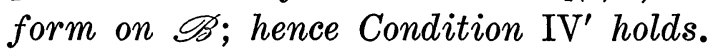

Proof. Choose $b_{0}>0$ so that III' holds, as was shown possible in in Lemma 6.1 ; then $H_{2}(e ; x)+b_{0} P_{2}(e ; x)$ is a Legendre form on $\mathscr{B}$ (Lemma 4.1). Since $\phi^{\sigma}[t, e(t)]=0(\sigma=1, \cdots, p)$, we see that $J_{2}(e ; x)=$ $H_{2}(e ; x)+b_{0} K(x)$ where $K(x)=2 \int_{T} \phi_{z_{\alpha}^{i}}^{\sigma} \phi_{z_{\beta} j}^{\sigma} x_{\alpha}^{i} x_{\beta}^{j} d t$. On $\mathscr{B}, K(x) \geqq 0$ and $\mathrm{IV}_{s}^{\prime}$ ensures that $H_{2}(x)>0$ whenever $K(x)=0$ and $x \neq 0$. Hence by the corollary of $\S 2$ there is a positive value of $b \geqq b_{0}$ such that $H_{2}(e ; x)+b K(x)$ is positive definite, which implies IV'.

It has been shown that Conditions $\mathrm{I}_{s}$, III', and $\mathrm{IV}_{s}^{\prime}$ imply Conditions $\mathrm{I}$, $\left[\mathrm{III} I^{\prime}\right.$, and $\mathrm{IV}^{\prime}$ of $\S 4$. Consequently Theorem 4.1 is applicable 
and has the following theorem as its interpretation in the case of a finite number of partial differential equations as side conditions:

THEOREM 6.1. If the function e as prescribed in the formulation of $\S 4$ satisfies Conditions $\mathrm{I}_{s}, \mathrm{III}_{s}^{\prime}$, and $\mathrm{IV}_{s}^{\prime}$ of this section, then there is a (weak) neighborhood $N$ of $e$ and a positive constant $\rho$ such that for every $z$ of class $\mathscr{A}$ in $N$

$$
H(z)-H(e)+c P(z) \geqq \rho\|z-e\|^{2} .
$$

$H(z)$ and $P(z)$ are given by (6.2) and (6.3), respectively.

COROLLARY 6.1. Under the hypotheses of Theorem 6.1, there is $a$ (weak) neighborhood $N$ of e and a positive constant $\rho$ such that for every $z$ of class $\mathscr{A}$ in $N$ the inequality

$$
H(z)-H(e) \geqq \rho\|z-e\|^{2}
$$

holds whenever $z$ also satisfies the conditions

$$
\phi^{\sigma}[t, z(t)]=0
$$$$
(\sigma=1, \cdots, p)
$$

7. A characterization of extremals, Let $J(z)$ be given by (3.3) and recall the assumptions and definitions of $\S 4$.

THEOREM 7.1. If the function $e$ is an extremal of $J(z)$ which satisfies the strengthened condition of Legendre, then there exists a finite set of functions $F^{\sigma}(t, z)(\sigma=1, \cdots, p)$ having the same continuity properties on $\mathscr{R}$ as $F(t, z)$, a (weak) neighborhood $N$ of $e$, and a positive constant $\rho$ such that for every $z$ of class $\mathscr{A}$ in $N$ the inequality $J(z)-J(e) \geqq \rho\|z-e\|^{2}$ holds whenever $z$ further satisfies the $p$ isoperimetric side conditions $J^{\sigma}(z)=\int_{T} F^{\sigma}(t, z) d t=0$. Moreover, the number $p$ can be taken equal to the isoperimetric index of $J_{2}(e ; x)$ on $T$.

Proof. We suppose given a function $e$ satisfying the hypotheses. For the choice of the side conditions, recall from $\S 2$ that there exist subspaces $\mathscr{B}_{+}, \mathscr{B}_{0}, \mathscr{B}_{-}$of $\mathscr{B}$ that are mutually orthogonal. $J_{2}$ is positive on $\mathscr{B}_{+}$, negative on $\mathscr{B}_{-}$, zero on $\mathscr{B}_{0}$ and $\mathscr{B}=\mathscr{B}_{-}+\mathscr{B}_{0}+\mathscr{B}_{+}$. $J_{2}$ is a Legendre form on $\mathscr{B}$ (Lemma 4.1), and so has a finite isoperimetric index. Therefore, let $y^{\sigma}(\sigma=1, \cdots, p)$ be a basis for $\mathscr{B}_{-}+\mathscr{B}_{0}$, and set $F^{\sigma}(t, z)=y_{\alpha}^{\sigma i}\left(z_{\alpha}^{i}-e_{\alpha}^{i}\right)$ (summed on $i \leqq n$ and $\alpha$, $|\alpha| \leqq k)$. Condition $\mathrm{I}_{s}$ is satisfied by the choices $a_{\sigma}=0(\sigma=1, \cdots, p)$, since $J_{1}(e ; x)=0$ by hypothesis; and the strengthened condition of Legendre ensures that $\mathrm{III}_{s}^{\prime}$ is satisfied. Condition $\mathrm{IV}_{s}^{\prime}$ reduces to 
considering $J_{2}(e ; x)$. Observing that $J_{1}^{\sigma}(e ; x)=\left(y^{\sigma}, x\right), J_{2}(e ; x)$ is positive for every $x$ such that $J_{1}(e ; x)=0$; therefore $\mathrm{IV}_{s}^{\prime}$ is also satisfied. The conclusion follows from Corollary 5.1.

The converse part of the characterization depends on proof of the necessity of the Condition $I_{s}$ of $\S 5$. This may be assumed as known from Mathis [13]. Other forms of isoperimetric conditions lead to the same result (cf. [1]).

\section{REFERENCES}

1. G. D. Birkhof and M. R. Hestenes, Natural isoperimetric conditions in the calculus of variations, Duke Math. J., 1 (1935), 198-286.

2. M. R. Hestenes, A sufficiency proof for isoperimetric problems in the calculus of variations, Bull. Amer. Math. Soc., 44 (1938), 662-667.

3. - Sufficient conditions for the isoperimetric problem of Bolza in the calculus of variations, Trans. Amer. Math. Soc., 60 (1946), 93-118.

4. — An indirect sufficiency proof for the problem of Bolza in non-parametric form, Trans. Amer. Math. Soc., 62 (1947), 509-535.

5. Sufficient conditions for multiple integral problems in the calculus of variations, Amer. J. of Math., 70 (1948), 239-276.

6. - Applications of the theory of quadratic forms in Hilbert space to the calculus of variations, Pacific J. of Math., 1 (1951), 525-581.

7. - Quadratic variational theory and linear elliptic partial differentiatl equations, Trans. Amer. Math. Soc., 101 (1961), 306-350.

8. A. R. Jacoby, Iterated integrals in the calculus of variations, $\mathrm{Ph}$. D. dissertation, Univ. of Chicago (1946), 38 pp.

9. W. E. Karush, $A$ semi-strong minimum for a multiple integral problem in the calculus of variations, Trans. Amer. Math. Soc., 63 (1948), 439-451.

10. - The index of an extremal arc, Canadian J. of Math., 4 (1952), 281-294.

11. R. Klotzler, Bemerkungen zu einigen Untersuchungen von M. I. Visik im Hinblick auf die Variationsrechnung mehrfacher Integrale, Math. Nachrichten, 17 (1958), 47-56.

12. E. J. McShane, Sufficient conditions for a weak relative minimum in the calculus of variations, Trans. Amer. Math. Soc., 52 (1942), 344-379.

13. H. F. Mathis, An isoperimetric problem for multiple integrals in the calculus of variations, Duke Math. J., 18 (1951), 177-184.

14. W. H. L. Myer, Multiple integral problems involving $n$ dependent functions in the calculus of variations, $\mathrm{Ph}$. D. dissertation, Univ. of Chicago (1947), $64 \mathrm{pp}$.

15. F. G. Myers, Sufficient conditions for the problem of Lagrange, Duke Math. J., 10 (1943), 73-97.

16. H. Poincaré, Sur les lignes geodesiques des surfaces convexes, Trans. Amer. Math. Soc., 6 (1905), 237-274.

17. W. T. Reid, Sufficient conditions by expansion methods for multiple integral problems of the calculus of variations, Bull. Amer. Math. Soc.. 45 (1939), 679.

18. L. Van Hove, Sur l'extension de la condition de Legendre du calcul des variations aux integrales multiples plusieurs fonctions inconnues, Indagationes Math., 9 no. 1 (1947), 3-8.

19. - Sur le signe de la variation seconde des integrales multiples a plusieurs fonctions inconnues, Acad. Roy. Belgique, Sci., Mem., 24 no. 5 (1949), 68 pp. 



\title{
PACIFIC JOURNAL OF MATHEMATICS
}

\author{
EDITORS
}

\author{
RoBerit OsSERman \\ Stanford University \\ Stanford, California
University of Washington
Seattle 5 , Washington \\ M. G. Arsove
}

\author{
J. DugundjI \\ University of Southern California
}

Los Angeles 7, California

Lowell J. Paige

University of California

Los Angeles 24, California

\section{ASSOCIATE EDITORS}
E. F. BECKENBACH
B. H. NeumanN
F. WOLF
K. YOSIDA

\section{SUPPORTING INSTITUTIONS}

\author{
UNIVERSITY OF BRITISH COLUMBIA \\ CALIFORNIA INSTITUTE OF TECHNOLOGY \\ UNIVERSITY OF CALIFORNIA \\ MONTANA STATE UNIVERSITY \\ UNIVERSITY OF NEVADA \\ NEW MEXICO STATE UNIVERSITY \\ OREGON STATE UNIVERSITY \\ UNIVERSITY OF OREGON \\ OSAKA UNIVERSITY \\ UNIVERSITY OF SOUTHERN CALIFORNIA
}

\author{
STANFORD UNIVERSITY \\ UNIVERSITY OF TOKYO \\ UNIVERSITY OF UTAH \\ WASHINGTON STATE UNIVERSITY \\ UNIVERSITY OF WASHINGTON \\ * * * * \\ AMERICAN MATHEMATICAL SOCIETY \\ CALIFORNIA RESEARCH CORPORATION \\ SPACE TECHNOLOGY LABORATORIES \\ NAVAL ORDNANCE TEST STATION
}

Mathematical papers intended for publication in the Pacific Journal of Mathematics should by typewritten (double spaced), and on submission, must be accompanied by a separate author's résumé. Manuscripts may be sent to any one of the four editors. All other communications to the editors should be addressed to the managing editor, L. J. Paige at the University of California, Los Angeles 24, California.

50 reprints per author of each article are furnished free of charge; additional copies may be obtained at cost in multiples of 50 .

The Pacific Journal of Mathematics is published quarterly, in March, June, September, and December. Effective with Volume 13 the price per volume (4 numbers) is $\$ 18.00$; single issues, $\$ 5.00$. Special price for current issues to individual faculty members of supporting institutions and to individual members of the American Mathematical Society: $\$ 8.00$ per volume; single issues $\$ 2.50$. Back numbers are available.

Subscriptions, orders for back numbers, and changes of address should be sent to Pacific Journal of Mathematics, 103 Highland Boulevard, Berkeley 8, California.

Printed at Kokusai Bunken Insatsusha (International Academic Printing Co., Ltd.), No. 6, 2-chome, Fujimi-cho, Chiyoda-ku, Tokyo, Japan.

PUBLISHED BY PACIFIC JOURNAL OF MATHEMATICS, A NON-PROFIT CORPORATION

The Supporting Institutions listed above contribute to the cost of publication of this Journal. but they are not owners or publishers and have no responsibility for its content or policies. 


\section{Pacific Journal of Mathematics}

\section{Vol. 14, No. $4 \quad$ August, 1964}

Homer Franklin Bechtell, Jr., Pseudo-Frattini subgroups . . . . . . . . . . . . . . . . 1129

Thomas Kelman Boehme and Andrew Michael Bruckner, Functions with convex means .............................................. 1137

Lutz Bungart, Boundary kernel functions for domains on complex manifolds . . . . . 1151

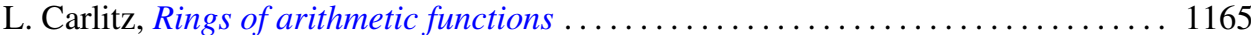

D. S. Carter, Uniqueness of a class of steady plane gravity flows . . . . . . . . . 1173

Richard Albert Dean and Robert Harvey Oehmke, Idempotent semigroups with

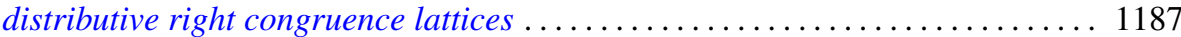

Lester Eli Dubins and David Amiel Freedman, Measurable sets of measures ..... . 1211

Robert Pertsch Gilbert, On class of elliptic partial differential equations in four

variables.......................................... 1223

Harry Gonshor, On abstract affine near-rings .................... 1237

Edward Everett Grace, Cut points in totally non-semi-locally-connected

continua ........................................... 1241

Edward Everett Grace, On local properties and $G_{\delta}$ sets ................ 1245

Keith A. Hardie, A proof of the Nakaoka-Toda formula . . . . . . . . . . . . . . . . 1249

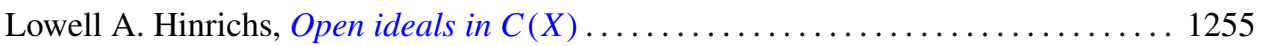

John Rolfe Isbell, Natural sums and abelianizing . . . . . . . . . . . . . . . . 1265

G. W. Kimble, A characterization of extremals for general multiple integral

problems............................................. 1283

Nand Kishore, A representation of the Bernoulli number $B_{n} \ldots \ldots \ldots \ldots \ldots \ldots \ldots \ldots$

Melven Robert Krom, A decision procedure for a class of formulas of first order predicate calculus.

Peter A. Lappan, Identity and uniqueness theorems for automorphic functions ...

Lorraine Doris Lavallee, Mosaics of metric continua and of quasi-Peano spaces . .

Mark Mahowald, On the normal bundle of a manifold..........

J. D. McKnight, Kleene quotient theorems

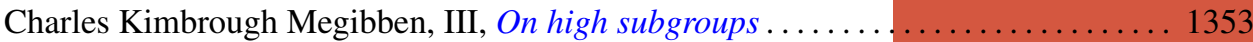

Philip Miles, Derivations on $B^{*}$ algebras . . . . . . . . . . . . . . . . . . . 1359

J. Marshall Osborn, A generalization of power-associativity . . . . . . . . . . . 1367

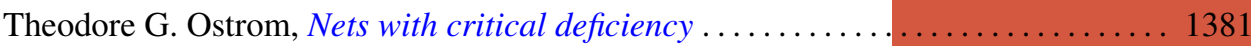

Elvira Rapaport Strasser, On the defining relations of a free product . . . . . . . . . 1389

K. Rogers, A note on orthoganal Latin squares . . . . . . . . . . . . . . . . . . 1395

P. P. Saworotnow, On continuity of multiplication in a complemented algebra ..... 1399

Johanan Schonheim, On coverings . . . . . . . . . . . . . . . . . . . . 1405

Victor Lenard Shapiro, Bounded generalized analytic functions on the torus . . . . . 1413

James D. Stafney, Arens multiplication and convolution . . . . . . . . . . . . . 1423

Daniel Sterling, Coverings of algebraic groups and Lie algebras of classical

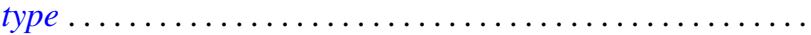

Alfred B. Willcox, Šlov type $C$ algebras over a connected locally compact abelian

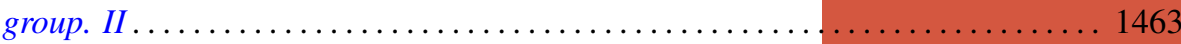

Bertram Yood, Faithful ${ }^{*}$-representations of normed algebras. II . . . . . . . . . 1475

Alexander Zabrodsky, Covering spaces of paracompact spaces 